\title{
CARACTERIZACIÓN Y VALORACIÓN BIOGEOGRÁFICA DE LOS PINARES DE PINUS UNCINATA DEL KARST DE LARRA (ALTO PIRINEO NAVARRO) PARA SU ORDENACIÓN Y GESTIÓN
}

\author{
Pedro José Lozano Valencia, José Antonio Cadiñanos Aguirre, \\ Itxaro Latasa Zeballos y Guillermo Meaza Rodriguez \\ Departamento de Geografía, Prehistoria y Arqueología de la UPV/EHU \\ joseantonio.cadinanos@ehu.es - pedrojose.lozano@ehu.es \\ guillermo.meatza@gmail.com - Itxaro.latasa@ehu.es
}

\begin{abstract}
Resumen: La presente aportación se enmarca en una línea de investigación en inventariación y valoración fitogeográfica iniciada hace más de 15 años y del que, de momento, es su último eslabón. Se trata de una metodología global de valoración paisajística centrado en la vegetación. Hasta la fecha, dicha propuesta se ha plasmado en diferentes trabajos (libros, artículos, capítulos de libro, comunicaciones y ponencias...) sobre algún territorio de la Península Ibérica y Chile. La metodología se aplica en esta ocasión al paisaje forestal de Pinus uncinata del Karst de Larra (Pirineo Navarro) con el objetivo de servir de herramienta valorativa para el diagnóstico de la calidad del medio con fines fundamentalmente conservacionistas.
\end{abstract}

Palabras clave: valoración biogeográfica, paisaje vegetal, Pinus uncinata, karst de Larra, Alto Pirineo Navarro.

\begin{abstract}
This paper has been written within the context of more than 15 years of research work and it is, for now, its last stage. It's a global landscape valuation method that focus on vegetation. To date, this methodology has been documented in numerous works (books, articles, chapters in books, documentation and lectures...) and it has been also applied for the study of some regions of the Iberian Peninsula and Chile. This methodology is now used for the study of Pinus uncinata dominated forest
\end{abstract}

Recibido: 2-05-13. Aceptado: 8-10-13. 
landscape of Larra Karst (navarrese Pyrenees). The aim is to serve as an assessment tool for the diagnosis of the quality of the environment for conservation purposes.

Key words: biogeographic valuation, plant landscape, Pinus uncinata, karst of Larra, High Pyrenees of Navarre.

\section{Introducción}

Una de las principales vertientes de la Biogeografía Aplicada es la valorativa, que trata de diagnosticar el estado actual del paisaje y la vegetación para su evaluación cualitativa. Desde esta perspectiva, es un importante instrumento en la ordenación y gestión territorial, una herramienta fundamental para el conocimiento y la toma de decisiones respecto a los paisajes vegetales considerados como patrimonio natural y cultural.

La presente aportación continúa una línea de investigación iniciada hace más de 15 años -siendo éste, de momento, su último eslabón-, que aborda una metodología global de inventariación y valoración paisajística centrado en la vegetación como elemento principal. Como lo atestiguan diversas referencias del apartado de bibliografía, hasta la fecha dicha propuesta se ha plasmado en diversos trabajos (libros, artículos, capítulos de libro, comunicaciones y ponencias...) y se ha aplicado a diversos de la Península Ibérica y Chile (Patagonia Chilena, Región Mediterránea Chilena). En esta ocasión se ha aplicado al paisaje forestal de Pinus uncinata del karst de Larra, que coloniza el piso subalpino del sector fronterizo entre la Comunidad Autónoma de Navarra y el Departamento Francés de Pirineos Atlánticos (Soule y Béarn).

La zona de estudio presenta unas características bien definidas, pues se trata de una región montañosa de latitudes medias, ubicada entre los 1.800 y los 2200 metros de altitud, sobre un sustrato rocoso calcáreo formado por calcarenitas y calizas con rudistas, denominadas también como "calizas de Larra". Estas litologías están sujetas a procesos de disolución muy activos por las copiosas precipitaciones y las bajas temperaturas. Como consecuencia, el paisaje es rico en todo tipo de morfologías exo (lapiaces cubiertos, semicubiertos y descubiertos, campos de dolinas, uvalas, etc.) y endokársticas (cuevas, simas...). A este respecto, es emblemática la afamada sima de la Piedra de San Martín, en el centro geográfico del territorio de estudio, una de las más profundas y amplias de Europa. El peculiar buzamiento de los estratos, junto con los activos procesos de disolución, ha dado lugar a una topografía anfractuosa de mogotes calcáreos que, a su vez, generan orientaciones y exposiciones muy contrastadas, factor determinante de las diferentes facies y subfacies del paisaje vegetal subalpino.

Las condiciones climáticas se corresponden al tipo Cfb de Köppen, subalpino, o subpirenaico en este caso. Se trata de un clima de inviernos largos y crudos, que hacen 


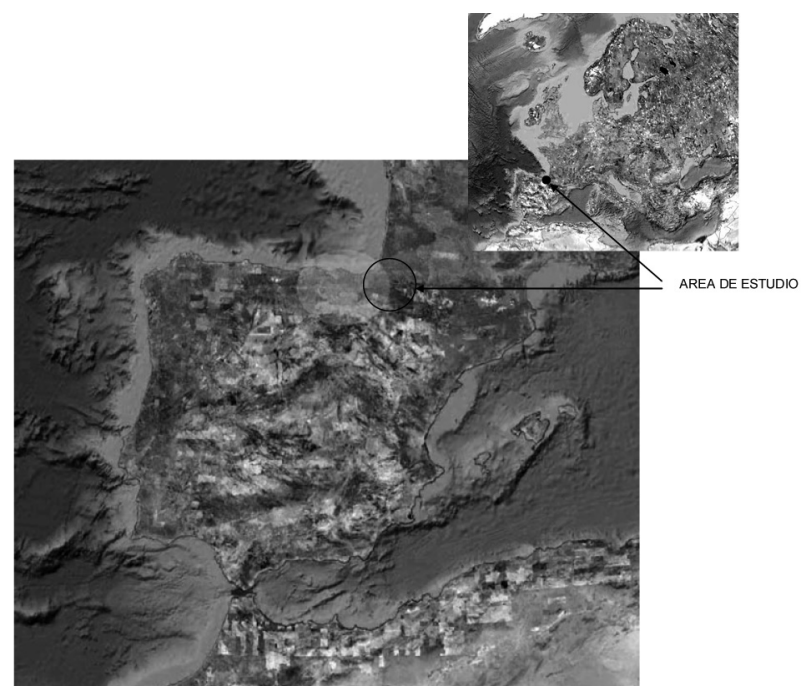

Figura 1. Localización del área de estudio.

Fuente: Elaboración propia.

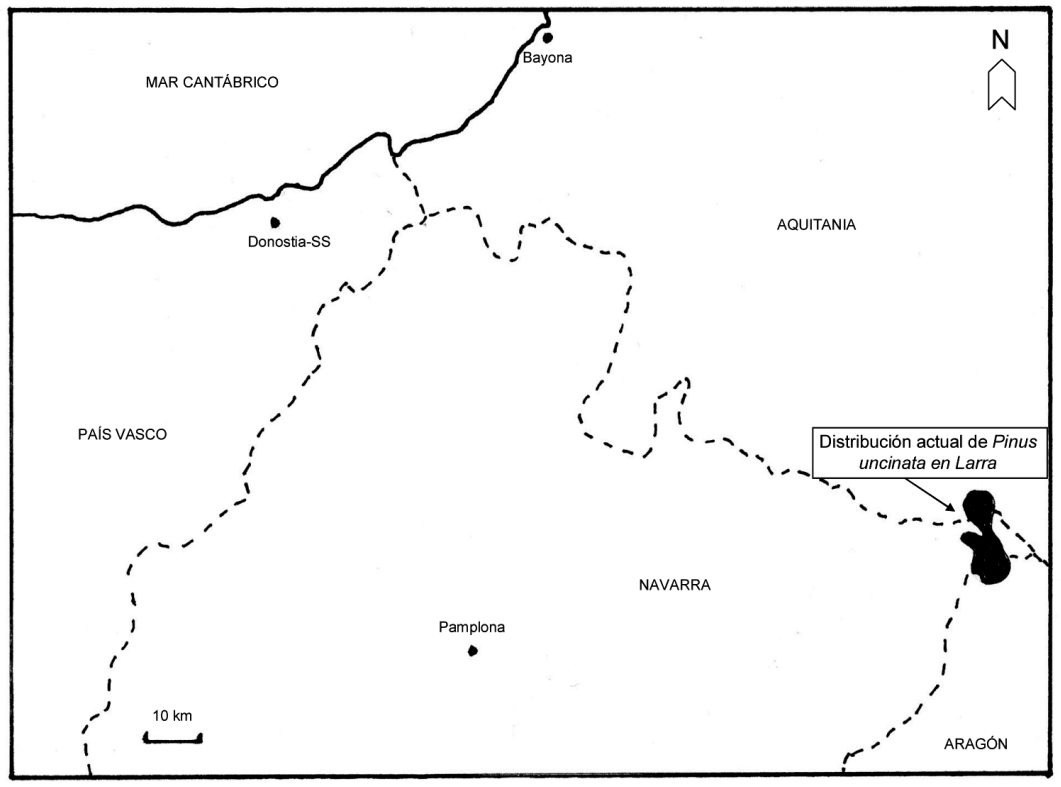

Figura 2. Distribución actual de Pinus uncinata en Larra.

Fuente: Elaboración propia. 
descender la temperatura media anual a menos de $7^{\circ} \mathrm{C}$. Las precipitaciones sobrepasan, a menudo con creces, los $2000 \mathrm{~mm}$ anuales, que se reparten de forma relativamente homogénea a lo largo del invierno, el otoño y la primavera, con un cierto declive en verano que se atenúa por efecto de las elevadas criptoprecipitaciones causadas por las nieblas. En verano y al final de la primavera, las precipitaciones caen en forma líquida y usualmente tormentosa, el resto del año son de nieve. En cualquier caso y pese a la relativa xericidad forzada por la facilidad de percolación del agua de lluvia en un sustrato kárstico, el nivel de humedad edáfica que requiere el bosque de pino negro ( $P i$ nus uncinata) está garantizado durante todo el año.

El generoso aporte de precipitaciones y su rápido circular hasta los sectores más profundos del sustrato dan lugar a un continuo lavado de los horizontes edáficos, de manera que, aunque el sustrato es eminentemente básico, el pH de los suelos tiende a ser neutro o ácido. De todos modos, salvo en algunas zonas llanas (fondos de dolinas, uvalas, fondos de cañones calizos...) donde puede sobrepasar metro y medio de potencia, en las laderas de fuerte pendiente y de lapiaz el suelo es esquelético o muy escaso y responde, de forma general, a un leptosol réndsico.

Aunque en el karst de Larra el territorio esté dominado por lo "mineral", el elemento vegetal adquiere una no menos notable potencialidad paisajística. Se trata de un cinturón subalpino dominado por el pinar de pino negro (Pinus uncinata), que encuentra su óptimo entre los 1700 y $2300 \mathrm{~m}$ de altitud y que ha sido profundamente modificado por la acción humana. El pinar de Larra configura, más que un auténtico ambiente nemoral, un matorral arbolado que mal cubre una topografía kárstica en extremo anfractuosa. El pino escala los resaltes más inaccesibles y aprovecha los suelos magros de las fisuras, lo que le obliga a crecer en grupos dispersos. Desde tiempos inmemoriales, este pinar, ya de por si ralo debido a las extremosas condiciones ambientales, se ha visto reducido por actividades seculares relacionadas fundamentalmente con la ganadería y la extracción de leña. Ello ha dado lugar a un bosque abierto que coloniza los sectores con menos suelo y mayor pendiente, mientras que en las zonas de topografía más amable y suelos más profundos el pinar ha sido eliminado en favor de una pradera subalpina que mantiene una ganadería tradicional y trashumante centrada en la oveja, la vaca pirenaica y determinadas razas caballares de gran resistencia a las condiciones montaraces. Llama poderosamente la atención el hecho de que se trate de un paisaje forestal reducido en extensión pero muy rico en elementos relictos, endémicos y finícolas, que encuentran en la zona de estudio su límite occidental y/o meridional de distribución.

\section{Objetivos}

El objetivo principal estriba en la aplicación de una metodología propia de inventariación y valoración del paisaje vegetal del Pirineo Occidental centrándonos, con- 
cretamente, en el diagnóstico de la calidad de los bosques de pino negro (Pinus uncinata) del karst de Larra (Pirineo Navarro).

Igualmente, se pretende cubrir otros dos objetivos operativos:

A) Consolidación de un modelo fácil y versátil de inventariación y valoración del paisaje vegetal, cara a la optimización de su eficacia en la más amplia gama de ámbitos territoriales.

B) Obtención de valoraciones parciales que puedan ser tenidas en cuenta de forma sectorial atendiendo a los atributos o cuestiones que se consideren oportunas a la hora de planificar y gestionar dicho paisaje, sus valores naturales, culturales, mesológicos, amenazas, etc.

\section{Metodología}

\subsection{Inventariación}

La primera fase de la propuesta metodológica se ha centrado en la recogida de datos sobre el terreno y la elaboración de los inventarios biogeográficos de las parcelas representativas de los diversos tipos de facies y subfacies vegetales. El modelo de inventario ha sido diseñado, ensayado, contrastado y corregido por los autores de este artículo en sucesivas aportaciones (Cadiñanos \& Meaza, 1998a, 1998b y 2000; Meaza, Cadiñanos \& Lozano, 2006). El objetivo primordial de este modelo de inventario es la recogida in situ de la mayor cantidad posible de datos geográficos y medioambientales necesarios para la posterior valoración biogeográfica del paisaje vegetal en cuestión. En primer lugar se obtienen los datos de localización e identificación del lugar (coordenadas UTM, topónimos, etc.), aspectos y rasgos geográficos y medioambientales generales (topográficos, litológicos, geomorfológicos, edáficos e hidrológicos), fotografías de la parcela, etc.

A continuación se identifica y anota cada una de las especies vegetales junto con su índice de cobertura, siguiendo el habitual sistema de notación fitosociológica. Además, se detalla, por un lado, su fisionomía biológica básica dividida en tres grupos (árboles y arbustos, matas y trepadoras y herbáceas); y, por otro, su presencia en 4 estratos verticales (superior, por encima de $5 \mathrm{~m}$; intermedio alto, 5-1 m; intermedio bajo, 1-0,5 m; e inferior, por debajo de $0,5 \mathrm{~m}$ ).

Se señala, asimismo, la presencia y densidad de briófitos (estrato muscinal), líquenes y hongos según el sustrato de crecimiento, simplificados en epífitos y terrícolassaxícolas, lo que resulta inexcusable para el cálculo de índices complementarios que 
matizan la riqueza en hábitats de la formación correspondiente. Salvo excepciones, no se entra en la determinación de los taxones concretos que conforman estas comunidades de talófitos.

Finalmente, se anota una serie de datos imprescindibles para la valoración complementaria de las comunidades forestales. Así, la cobertura global y la riqueza por estratos (COBEST y RIQUEST), la diversidad de hábitats y sinusias no desglosables a la escala de trabajo (FORHAB), la superficie de la mancha homogénea (FORESP), la variedad dasonómica tipológica (FORFIS) y los valores patrimoniales, culturales y etnográficos añadidos (FORPAT).

Como es fácil advertir, estos inventarios son más procelosos y requieren más tiempo que los fitosociológicos o similares; pero, una vez realizados, la información que contienen es cuantitativa y cualitativamente superior, lo que permite su posterior utilización no sólo para la caracterización geobotánica de la zona en cuestión, sino también para reflejar su disposición estructural y biogeográfica así como, en última instancia, la evaluación y gestión de la vegetación, fauna y paisaje.

En el caso concreto de los pinares de Pinus uncinata del karst de Larra, tras un dilatado trabajo de campo que se inició en 1990, se han caracterizado y evaluado un total de 27 inventarios. A lo largo de las diferentes estancias y en el marco de varios proyectos de investigación y con el fin de evitar sesgos, se hicieron repetidas visitas en diferentes momentos fenológicos.

A partir de dichos inventarios se han determinado dos grandes facies forestales de Pinus uncinata: la de pinar de pino negro en umbría, y la de pinar de pino negro en solana. Dentro de cada una de ellas puede diferenciarse, a su vez, dos subfacies en relación a su riqueza en especies. De esta manera, se han discernido cuatro subunidades, caracterizadas por sus correspondientes sininventarios florísticos. Es posible reconocer una quinta subfacies, que respondería a un pinar instalado en una topografía llana y, por lo tanto, orientada a todos los vientos, bastante pobre y pareja florísticamente a la de umbría.

He aquí las cinco subfacies:

- Pinar de pino negro (Pinus uncinata) en solana:

1. Subfacies rica en cobertura vegetal.

2. Subfacies pobre en cobertura vegetal.

- Pinar de pino negro (Pinus uncinata) en umbría:

3. Subfacies rica en especies y cobertura vegetal.

4. Subfacies pobre en especies y cobertura vegetal.

5. Pinar de pino negro en exposición a todos los vientos (facies única) 
Tabla 1. Ejemplo de inventario biogeográfico

INVENTARIO PARA VALORACIÓN DE LA VEGETACIÓN № LAR21

FECHA: 02/05/2006-15/09/2010-04/07/2012

Pinar de Pino negro abierto con gayuba

\begin{tabular}{|c|c|c|c|c|c|c|}
\hline & TAXONES \Según estratos en metros & $>5$ & 5 a $1 \mathrm{~m}$. & $1-0,5$ & $<0,5$ & global \\
\hline \multirow{4}{*}{ 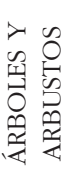 } & Pinus uncinata & 3 & 1 & 1 & 1 & 4 \\
\hline & Sorbus intermedia & - & + & + & - & + \\
\hline & Sorbus aucuparia & - & - & - & + & + \\
\hline & Rhamnus alpinus & - & - & - & + & + \\
\hline \multirow{6}{*}{ 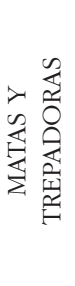 } & Juniperu communis ssp. alpina & - & - & 1 & 1 & 2 \\
\hline & Arctostaphylos uva-ursi & - & - & - & 2 & 2 \\
\hline & Genista occidentalis & - & - & - & 2 & 2 \\
\hline & Daphne laureola & - & - & - & 1 & 1 \\
\hline & Calluna vulgaris & - & - & - & + & + \\
\hline & Lonicera pyrenaica & - & - & - & + & + \\
\hline \multirow{20}{*}{ 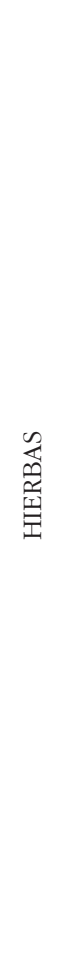 } & Festuca gautieri & & & & 2 & 2 \\
\hline & Helleborus viridis & & & & 1 & 1 \\
\hline & Teucrium pyrenaicum & & & & 1 & 1 \\
\hline & Gallium pinetorum & & & & 1 & 1 \\
\hline & Scilla verna & & & & 1 & 1 \\
\hline & Gentiana occidentalis & & & & 1 & 1 \\
\hline & Viola riviniana & & & & 1 & 1 \\
\hline & Thymus praecox & & & & + & + \\
\hline & Polystichum aculeatum & & & & + & + \\
\hline & Erinus alpinus & & & & + & + \\
\hline & Saxifraga paniculata & & & & + & + \\
\hline & Pilosella sp. & & & & + & + \\
\hline & Hepatica nobilis & & & & + & + \\
\hline & Polygala alpestris & & & & + & + \\
\hline & Asplenium trichomanes & & & & + & + \\
\hline & Heliamthemum oelandicum & & & & + & + \\
\hline & Taraxacum dissectum & & & & + & + \\
\hline & Carex ornithopoda & & & & + & + \\
\hline & Avenula pratensis & & & & + & + \\
\hline & Teucrium chamaedrys & & & & + & + \\
\hline
\end{tabular}




\begin{tabular}{|c|c|c|c|c|c|c|c|}
\hline & \multicolumn{2}{|c|}{ TAXONES \Según estratos en metros } & $>5$ & 5 a $1 \mathrm{~m}$ & $1-0,5$ & $<0,5$ & global \\
\hline 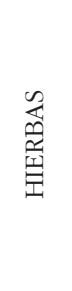 & \multicolumn{2}{|c|}{$\begin{array}{l}\text { Thymelaea dioica } \\
\text { Asplenium ruta-muraria } \\
\text { Galium saxatile } \\
\text { Hieracium gr. Murorum } \\
\text { Arabis ciliata } \\
\text { TOTAL }\end{array}$} & 3 & 1 & 1 & $\begin{array}{l}+ \\
+ \\
+ \\
+ \\
+ \\
3\end{array}$ & $\begin{array}{l}+ \\
+ \\
+ \\
+ \\
+\end{array}$ \\
\hline 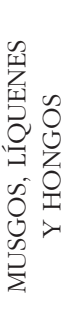 & \multicolumn{2}{|c|}{$\begin{array}{l}\text { Líquenes ligados a troncos y ramas } \\
\text { Líquenes ligados a suelo y rocas } \\
\text { Musgos en troncos } \\
\text { Musgos en rocas y suelos } \\
\text { Hongos } \\
\text { Hojarasca } \\
\text { Suelo o roca desnuda }\end{array}$} & & & & $\begin{array}{l}1 \\
+ \\
+ \\
+ \\
+ \\
+ \\
2\end{array}$ & \\
\hline \multicolumn{3}{|c|}{ Cobertura global por estrato } & 3 & 1 & 1 & 3 & 8 \\
\hline \multicolumn{2}{|c|}{ RIQUEST } & $\begin{array}{l}n^{o} \text { especies } \\
\text { por estrato } \\
\text { puntuación }\end{array}$ & 1 & $\begin{array}{l}2 \\
2\end{array}$ & 3 & 34 & $\begin{array}{l}35 \\
10\end{array}$ \\
\hline
\end{tabular}

Fuente: Elaboración propia.

\subsection{Valoración}

La propuesta de valoración, que ofreció sus primeros resultados hace ya 20 años (Meaza, 1993), y que ha adquirido su conformación actual tras la empiria de aplicación a muy diversos territorios y paisajes, pretende ofrecer una metodología coherente, rigurosa, versátil y práctica, basada en pautas sencillas, flexibles y claras, con resultados estándar fáciles de aplicar e interpretar de cara a una correcta y jerárquica gestión de los paisajes vegetales concernidos.

Como puede apreciarse en la figura 2, el método descansa en conceptos valorativos diferenciados que constituyen, al tiempo, eslabones estrechamente ligados del sistema operativo (Cadiñanos y Meaza, 2000, para la metodología general; Cadiñanos y Meaza, 2000, para la metodología específica forestal). 


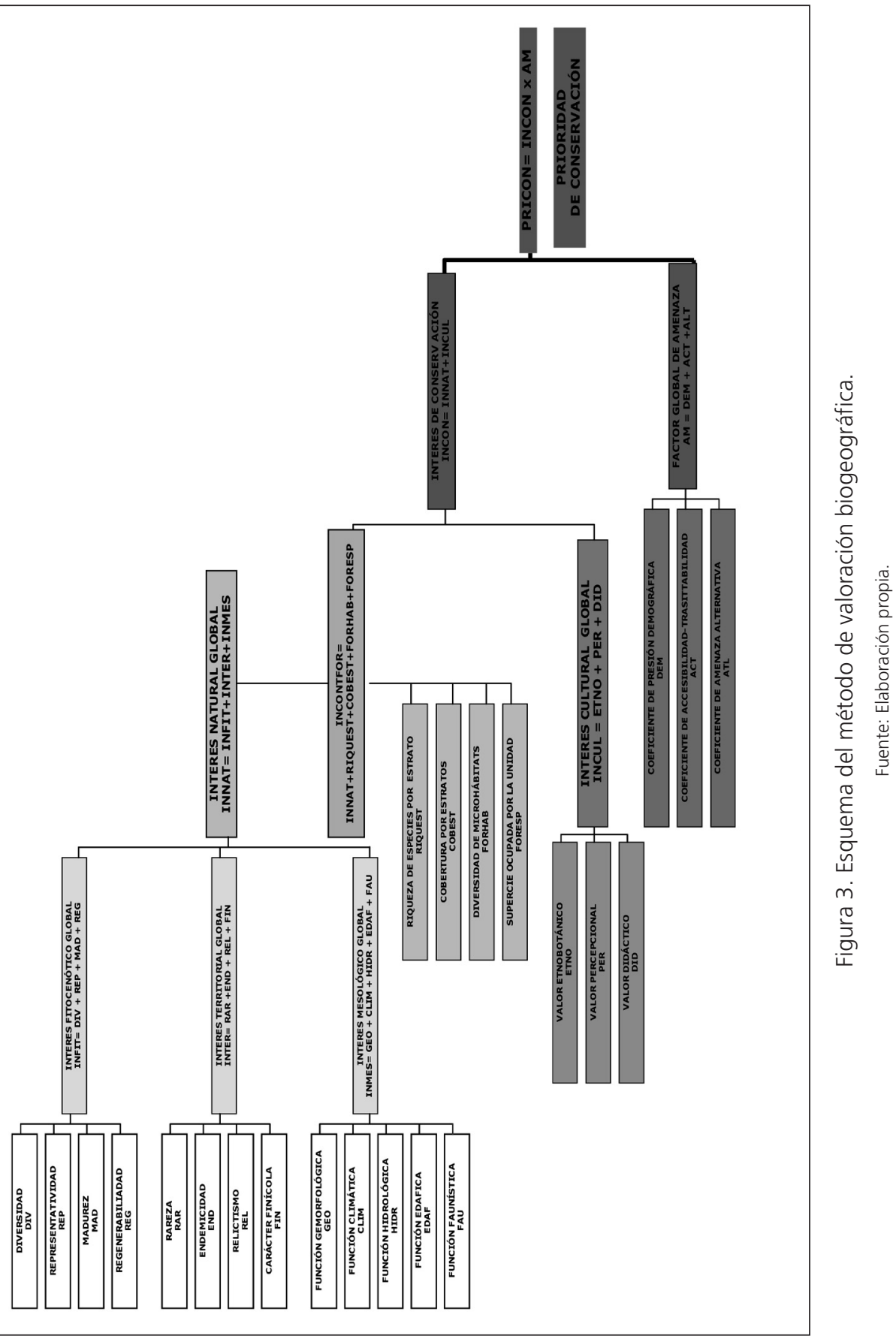




\subsubsection{Interes Fitocenótico Global (INFIT)}

Los criterios fitocenóticos estiman caracteres intrínsecos a la vegetación y el paisaje, tales como la diversidad, representatividad, madurez y regenerabilidad espontánea. Cada índice puede valorarse de 1 a 10 puntos dependiendo de la calidad o cantidad que muestre; no obstante, atendiendo a su mayor importancia relativa, el índice de madurez es multiplicado por el factor de corrección 2. Como consecuencia, el INFIT de cada unidad puede variar entre 5 y 50 puntos siguiendo la siguiente fórmula:

INFIT = DIV (1 a 10) + REP (1 a 10) + MAD (2 a 20) + REG (1 a 10).

\subsubsection{Interés Territorial Global (INTER)}

Los criterios territoriales son bifactoriales (se aplican tanto a nivel de especie como de agrupación) y consideran los atributos de rareza, endemismo, relictismo y carácter finícola, tanto de los taxones presentes como de la propia formación o unidad de paisaje. Cada índice puede valorarse de 0 a 10 puntos dependiendo de la calidad que muestre; no obstante, el índice de rareza es multiplicado por un factor de corrección 2 , en coherencia con su mayor importancia relativa. Así, la unidad valorada puede obtener un INTER que varía entre 0 y 50 puntos siguiendo la siguiente fórmula:

$$
\text { INTER }=\text { RAR }(0 \text { a 20) + END }(0 \text { a 10) + REL (0 a 10) + FIN (0 a 10). }
$$

\subsubsection{Interés Mesológico Global (INMES)}

Los criterios mesológicos evalúan la contribución de la vegetación a la protección, equilibrio y estabilidad de la zoocenosis, el hábitat y el geo-biotopo en el que radica. En su virtud, se proponen 5 parámetros, correspondientes a las funciones geomorfológica, climática, hidrológica, edáfica y faunística, todos ellos estrecha y complejamente relacionados. Cada índice puede valorarse de 1 a 10 puntos dependiendo de su nivel de calidad; en este caso y en consonancia con su mayor importancia relativa, es el índice de función geomorfológica el multiplicado por un factor de corrección 2. La unidad valorada obtiene un INMES que puede variar entre 6 y 60 puntos siguiendo la siguiente fórmula:

$$
\begin{gathered}
\text { INMES }=\text { GEO }(2 \text { a } 20)+\operatorname{CLIM}(1 \text { a } 10)+\operatorname{HIDR}(1 \text { a } 10)+ \\
\text { EDAF }(1 \text { a } 10)+\text { FAU }(1 \text { a } 10) .
\end{gathered}
$$


La suma de estos tres criterios da lugar al denominado Interés Natural Global (INNAT) y, por tanto, puede ser utilizado como criterio de raigambre y naturaleza puramente ambiental. En cualquier caso, dicho parámetro puede oscilar entre los 11 y 110 puntos. Su fórmula sería:

INNAT $=$ INFIT $(5$ a 50) + INTER (0 a 50) + INMES (6 a 60).

\subsubsection{Valoración de la Riqueza de especies por estrato (RIQUEST)}

Este criterio evalúa la mayor o menor diversidad de especies de cada uno de los estratos. Se utiliza la siguiente escala de valores: 1 punto para el estrato monoespecífico, 2 para el que cuenta con 2 a 4 taxones, 3 para el que tiene 5 a 9, 4 para el que presenta 10 a 19 y, por último, 5 para el que contiene más de 20 taxones. Se suman las puntuaciones parciales de los distintos estratos y se multiplica por 0,5. De esta forma, este índice puede fluctuar entre 2 y 10 puntos.

\subsubsection{Valoración de la Cobertura por estrato (COBEST)}

Se trata de un criterio que valora la mayor o menor cobertura de cada uno de los estratos. Se aplica la siguiente escala de valores: 1 punto para el estrato con cobertura +ó 1, 2 para aquel que cuenta con cobertura 2, 3 para el que tiene 3, 4 para el que presenta 4 y, por último, 5 para el de 5 . Se suman las puntuaciones parciales de los distintos estratos y se multiplica por 0,5. De esta forma, este índice puede fluctuar entre 1 y 25 puntos.

\subsubsection{Valoración de la Diversidad de microhábitats o cualidades de diversidad (FORHAB)}

Este criterio evalúa la mayor o menor riqueza, dentro del dominio del sininventario, de diferentes microambientes o cualidades relacionadas con la biodiversidad de otros elementos, tales como líquenes, hongos, musgos, etc. El valor máximo no puede superar, por convención, los 20 puntos. Se otorga un punto por cada uno de los siguientes conceptos: existencia de hábitats acuáticos lénticos diferenciados; hábitats acuáticos lóticos y no esporádicos; hábitats semiacúaticos; hábitats rupícolas; hábitats hipógeos o semihipógeos; abundancia o diversidad de líquenes epífitos o terrícolas; abundancia o diversidad de musgos y briofitos; abundancia o diversidad de hongos; por la presencia de algunos troncos añosos vivos o muertos en pie; por la abundancia de troncos y ramas muertas en suelo; por cualquier otro aspecto positivo para la diversidad del bosque no contemplado anteriormente. 


\subsubsection{Valoración de la Superficie homogénea ocupada por la comunidad (FORESP)}

Se trata de una variable espacial. Este concepto se ha tomado de KIRBY (1986) que intenta, mediante este parámetro, valorar los paisajes vegetales más vastos a la vez que penalizar los menos amplios, a causa del conocido "efecto oasis". Sin embargo, dado lo confuso de la formulación original de dicho autor, hemos optado por la siguiente escala:

1 punto por cada 0’4 ha compactas de bosque, hasta 2 puntos máximo.

1 punto por cada 1 ha compacta de bosque, hasta 3 puntos máximo.

1 punto por cada 4 ha compactas de bosque, hasta 2 puntos máximo.

1 punto por cada 10 ha compactas de bosque, hasta 9 puntos máximo.

2 puntos por cada 100 ha compactas de bosque, hasta 18 puntos máximo.

4 puntos por cada 1000 ha compactas de bosque (sin máximo).

La suma del INNAT, más la riqueza de especies por estrato, más la cobertura por estratos, más la diversidad de microhábitats, más la valoración de la superficie homogénea ocupada por la comunidad da lugar al INCONTFORT. Donde aparecen reflejados, además de los valores naturales, otros determinados por la estructura y riqueza en taxones de la unidad, los diferentes microámbientes que se puedan encontrar, así como la extensión de la mancha homogénea.

INCONTFOR = INNAT $(11$ a 110) + RIQUEST $(2$ a 10) + COBEST $(1$ a 25$)+$ FORHAB (0 a 20) + FORESP (0 a X).

\subsubsection{Valoración del Interés Cultural (INCUL)}

Los criterios de carácter cultural han sido obviados o infrautilizados en la mayor parte de las propuestas valorativas debido, básicamente, al reduccionismo naturalístico de la generalidad de los expertos provenientes del campo de las Ciencias Naturales, y/o a unas dificultades de objetivación sin duda más serias que las que plantean los parámetros de carácter natural. Sin embargo, concitan una atención cada día mayor en la sensibilidad y políticas conservacionistas. El INCUL se calcula teniendo en cuenta, a su vez, tres valores diferentes.

\subsubsection{Valor Etnobotánico (ETNO)}

Este criterio trata de evaluar los aspectos etnoculturales (históricos, arqueológicos, religiosos, mitológicos, simbólicos, recreativos, medicinales...) de las plantas, la vegetación y el paisaje que, en su caso, pueden contribuir a hacerlas acreedoras de conser- 
vación. Cabría destacar, entre todos ellos, los de tipo más directamente etnográfico o ligados a "modos de vida" ancestrales: elementos simbólicos (árboles o bosques singulares ligados a la cultura tradicional), vestigios, estructuras y microtopografías relictuales de prácticas forestales (morfología de fustes y ramaje; muros, lezones, setos, caballones y cárcavas de contención o de separación de parcelas; carboneras...), agroganaderas o preindustriales (ferrerías, molinos, aceñas, batanes...) configuradoras de paisajes vegetales peculiares. Se recomienda adjudicar 1 punto por cada elemento considerado de alto valor etnobotánico, respetando siempre la escala de 1 a 10 puntos. El ETNO es multiplicado por un factor de corrección de 2 puesto que cuenta con mayor importancia que los otros tres, de manera que puede fluctuar entre 0 y 20 puntos.

\subsubsection{Valor Percepcional (PER)}

Es un parámetro que trata de valorar la relación perceptiva (escénica, estética, incluso vivencial) del hombre con respecto a la vegetación. Para su correcta evaluación, lo ideal es contar con encuestas objetivas de preferencias, gustos, querencias y afinidades. El PER fluctúa entre 1 y 10 puntos.

\subsubsection{Valor Didáctico (DID)}

Criterio que trata de aquilatar el interés pedagógico del paisaje en sus aspectos naturales y culturales y en la educación y concienciación ambiental de la población en general. Se propone la utilización de la siguiente escala genérica que el investigador habrá de aplicar con el mejor criterio posible: desde el valor 1 a aquellas unidades que cuenten con un valor didáctico muy bajo hasta el 10 a aquellas que obtengan uno de muy alta estima. Para ello también se pueden valorar cuestiones que, en los últimos tiempos, han gozado de un gran desarrollo y que influyen en la sensibilización, concienciación, educación y formación (existencia de senderos marcados, carteles explicativos, rutas montañeras, estaciones de observación, aulas de naturaleza, centros de interpretación, etc.). En el medio aparecerán las diferentes gradaciones, bajo, medio y alto. De esta forma el DID puede fluctuar entre 1 y 10 puntos.

En definitiva, el Interés Cultural (INCUL) deriva de la suma de las calificaciones adjudicadas a los 3 criterios valorativos que lo integran. Esto es:

$$
\text { INCUL }=\text { ETNO }(2 \text { a } 20)+\text { PER }(1 \text { a } 10)+\text { DID }(1 \text { a } 10)
$$

El interés cultural global oscila, entonces, entre los 4 y 40 puntos. 


\subsubsection{El interés de conservación de una determinada agrupación vegetal o paisaje (INCON)}

Resulta de sumar a la puntuación de INNAT (11 a 160) la calificación obtenida por INCUL ( 4 a 40), con lo que el rango de INCON oscila entre 15 y 200 puntos.

\subsubsection{La Prioridad de conservación (PRICON)}

Este concepto es solidario pero, al tiempo, sustancialmente diferente al de INCON (interés de conservación), ya que incluye consideraciones ajenas, extrínsecas, a este último. Su resultado ha de ser asumido de manera independiente y no debe ser confundido con él. En efecto, la necesidad de evaluar de forma independiente la prioridad de conservación es corolario de una interrogante insoslayable: ¿Puede un espacio de menor interés de conservación que otro requerir, pese a ello, una mayor urgencia de protección por estar más amenazado? La respuesta parece clara: sí, pero siempre que su interés de conservación sea suficientemente alto. La fórmula que se propone para hallar este valor es la de multiplicar INCON por el factor de amenaza. La prioridad de conservación está, pues, expresamente ideada para su utilización por la administración competente o el gestor, quienes precisan de un diagnóstico claro y operativo sobre cuáles son los espacios que deben ser priorizados cara a su protección y cuáles pueden esperar.

El grado de amenaza que pesa sobre las unidades de vegetación o paisajes concernidos en el proceso evaluativo se calibra en función de tres parámetros: presión demográfica, accesibilidad-transitabilidad y amenaza alternativa.

\subsubsection{Coeficiente de Presión Demográfica (DEM)}

Introduce la variable demográfica humana en el sistema valorativo. En su virtud, se priman o penalizan situaciones de alta o baja densidad de población, con mayor o menor peligro, respectivamente, de alteración de la vegetación. La escala a aplicar se obtiene en función de los rangos de densidad real en habitantes $/ \mathrm{km}^{2}$ de la zona de estudio. El investigador debe tener en cuenta cuestiones como la demografía de la zona, cercanía a grandes núcleos de población y conurbaciones y flujos poblacionales estacionales, así como la disponibilidad y nivel de detalle de las fuentes estadísticas. La escala propuesta varía entre el 1 para aquellos ámbitos con densidades de menos de 50 habitantes por $\mathrm{km}^{2}$ hasta 10 en aquellos que se superen los $450 \mathrm{Hab} / \mathrm{km}^{2}$. 


\subsubsection{Coeficiente de Accesibilidad-Transitabilidad (ACT)}

Es un parámetro de atención inexcusable a la hora de establecer el nivel de amenaza al que se encuentra expuesta la unidad de paisaje, puesto que la presencia e impronta del ser humano está condicionada por la topografía del terreno, la densidad, tamaño, estado de conservación y grado de penetración de la red viaria y por la estructura más o menos abierta de la unidad valorada; en su caso, también por las limitaciones impuestas por los propietarios o administradores del terreno o por normativa legal dictada por la Administración. La escala propuesta muestra una matriz de doble entrada -6 valores de accesibilidad y otros 6 de transitabilidad desde muy baja hasta absoluta para las dos-. La combinación de las dos variables va generando puntuaciones que varían desde el 1 hasta 10 cuando la accesibilidad y transitabilidad son absolutas.

\subsubsection{Coeficiente de Amenaza Alternativa (ALT)}

Se incluyen y calibran bajo este concepto factores alternativos de amenaza que, eventualmente, puedan afectar a la unidad de vegetación o paisaje objeto de evaluación de manera grave, real y coetánea al ejercicio valorativo -o a muy corto plazo-: catástrofes naturales o provocadas (inundaciones, fuegos), daños palpables por lluvia ácida, vertidos tóxicos o contaminantes, eutrofización, plagas u otras causas de mortalidad excesiva, invasión o desplazamiento de la vegetación original por plantas xenófitas agresivas, desaparición de la vegetación a corto plazo por talas masivas, acondicionamiento para infraestructuras, construcciones, tendidos eléctricos, depósitos, dragados, actividades extractivas, etc. La escala propuesta varía desde el 1 para la amenaza alternativa muy baja hasta el 10 para la muy alta.

Así, una vez obtenidos los tres coeficientes se obtiene el Factor Global de Amenaza (AM) sumando los valores de los coeficientes demográfico (DEM = 1-10), de accesibilidad-transitabilidad (ACT $=1-10)$ y de amenaza alternativa (ALT $=1-10)$, con lo que el resultado de AM oscila entre 3 y 30 puntos.

\subsubsection{La prioridad de conservación (PRICON)}

De una determinada agrupación vegetal o paisaje se determina multiplicando su valor de INCON (88 a 200) por el coeficiente AM (3 a 30) que le corresponda, con lo que el rango de PRICON oscila entre 264 y 6000 puntos. 
Tabla 2. Sininventarios de los pinares de Pinus uncinata del Karst de Larra (Pirineo Navarro)

\begin{tabular}{|c|c|c|c|c|c|c|}
\hline & TAXONES & & & & & \\
\hline \multirow{7}{*}{ 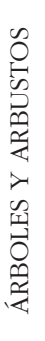 } & Pinus uncinata & 2 & 4 & 4 & 2 & 4 \\
\hline & Sorbus intermedia & 1 & + & 1 & + & + \\
\hline & Sorbus aucuparia & 1 & + & 1 & + & + \\
\hline & Sorbus chamaemespilus & + & & & & \\
\hline & Fagus sylvatica & + & & + & & + \\
\hline & Ramnus alpinus & & + & + & 1 & \\
\hline & Sorbus aria & & & & & + \\
\hline \multirow{15}{*}{ 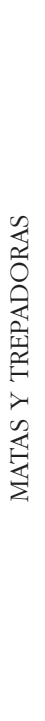 } & Rododendron ferrugineum & 4 & & 4 & & \\
\hline & Vaccinium myrtillus & 3 & & 3 & 1 & 3 \\
\hline & Vaccinium uliginosum & 2 & & 1 & & \\
\hline & Calluna vulgaris & 2 & + & 2 & & \\
\hline & Salix pyrenaica & + & & + & & \\
\hline & Juniperus communis subsp. alpina & + & & 2 & & \\
\hline & Amelanchier ovalis & + & & & + & \\
\hline & Daphne laureola & + & 1 & 2 & + & 1 \\
\hline & Juniperus alpina & & 2 & & 4 & 1 \\
\hline & Arctostaphyloss wva-ursi & & 2 & + & 3 & 1 \\
\hline & Genista occidentalis & & 2 & + & & \\
\hline & Lonicera pyrenaica & & + & & & \\
\hline & Rosa pendulina & & & 1 & 1 & \\
\hline & Erica vagans & & & + & & \\
\hline & Ribes alpinus & & & & + & \\
\hline \multirow{11}{*}{ 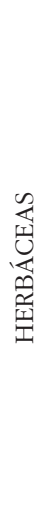 } & Festuca gautieri & 2 & 2 & 2 & 3 & 5 \\
\hline & Scilla verna & 1 & 1 & + & & + \\
\hline & Luzula nutans & 1 & & & & \\
\hline & Melampyrum pratense & 1 & & + & & \\
\hline & Alchemilla gr. plicatula & 1 & & 1 & & + \\
\hline & Thymus praecox polytrichus & 1 & + & + & & \\
\hline & Hieracium lactucella & 1 & & & & \\
\hline & Avenula pratensis & 1 & + & & + & + \\
\hline & Pinguicula grandiflora & 1 & & & & \\
\hline & Lotus curniculatus & + & & & & \\
\hline & Pilosella sp. & + & + & + & + & + \\
\hline
\end{tabular}




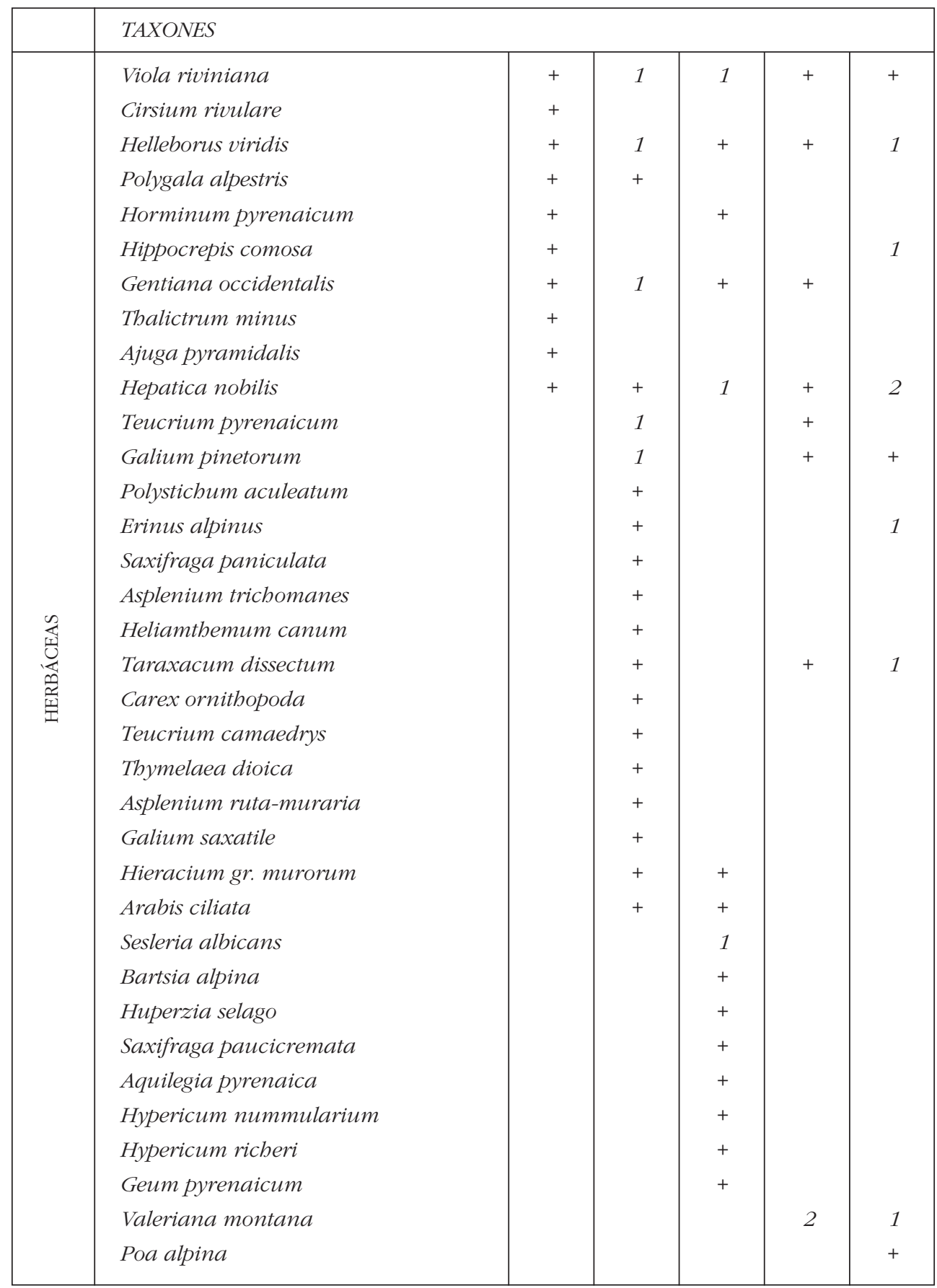




\begin{tabular}{|c|c|c|c|c|c|c|}
\hline & TAXONES & & & & & \\
\hline \multirow{7}{*}{ 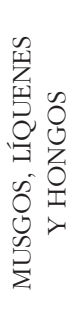 } & Líquenes ligados a troncos y ramas & 2 & 1 & 2 & 1 & 1 \\
\hline & Líquenes ligados a suelo y rocas & 2 & + & 2 & + & 1 \\
\hline & Musgos en troncos & 1 & + & 1 & + & 1 \\
\hline & Musgos en rocas y suelos & 1 & + & 1 & + & 1 \\
\hline & Hongos & & & & & \\
\hline & Hojarasca & 1 & + & 1 & + & 1 \\
\hline & Suelo o roca desnuda & 2 & 2 & 2 & 2 & 3 \\
\hline \multicolumn{2}{|c|}{ № Total de taxones } & 34 & 35 & 37 & 22 & 23 \\
\hline
\end{tabular}

Fuente: Elaboración propia.

\section{Resultados y discusión}

En la tabla 3 aparecen las diferentes coberturas registradas para cada uno de los estratos de vegetación valorados por cada uno de los cinco sininventarios.

Tabla 3. Coberturas generales de los 4 estratos de los diferentes sininventarios de los pinares de Pinus uncinata del Karst de Larra (Pirineo Navarro)

\begin{tabular}{|c|c|c|c|c|}
\hline Cobertura global por estratos & $>5 m$ & $5 m a 1 m$. & $1 m-0,5 m$ & $<0,5 m$ \\
\hline 1 & 2 & 1 & 2 & 4 \\
2 & 3 & 1 & 1 & 3 \\
3 & 2 & 1 & 2 & 4 \\
4 & 3 & 1 & 1 & 3 \\
5 & 4 & + & + & 5 \\
\hline
\end{tabular}

Fuente: Elaboración propia.

El pinar de Pinus uncinata de Larra configura, más que un auténtico ambiente nemoral, un matorral arbolado que mal cubre una topografía kárstica en extremo anfractuosa. El pino escala los resaltes más inaccesibles y aprovecha los suelos magros de las fisuras, lo que le obliga a crecer en grupos dispersos. Los contados individuos que superan la etapa de retoño han de hacer frente a circunstancias extremadamente hostiles: fuertes heladas, alta radiación solar, tormentas con gran aparato eléctrico -abundan los pinos calcinados-, vientos huracanados (copas en bandera), inestabilidad del 
terreno (acodamiento y traza tortuosa del fuste), sobrepeso de nieve (serias mutilaciones), pérdida constante de suelo... e intensa explotación antrópica.

Se trata, pues, de un bosque abierto con aspecto, a menudo, más de matorral salpicado de árboles que de una verdadera foresta, lo que, de partida, es achacable a su crecimiento en un ambiente de lapiaces, cantiles y roquedos. La cobertura general del único árbol que puede sobrepasar, de forma generalizada, los 5 metros de porte -el propio $\mathrm{Pi}$ nus uncinata- oscila entre algo menos del $25 \%$ para los sininventarios 1 y 3 y algo más del 50\% para el sininventario 5. Esto hacía presuponer que la aplicación de algunos aspectos de la valoración forestal de tipo espacial iba a resultar un tanto problemática.

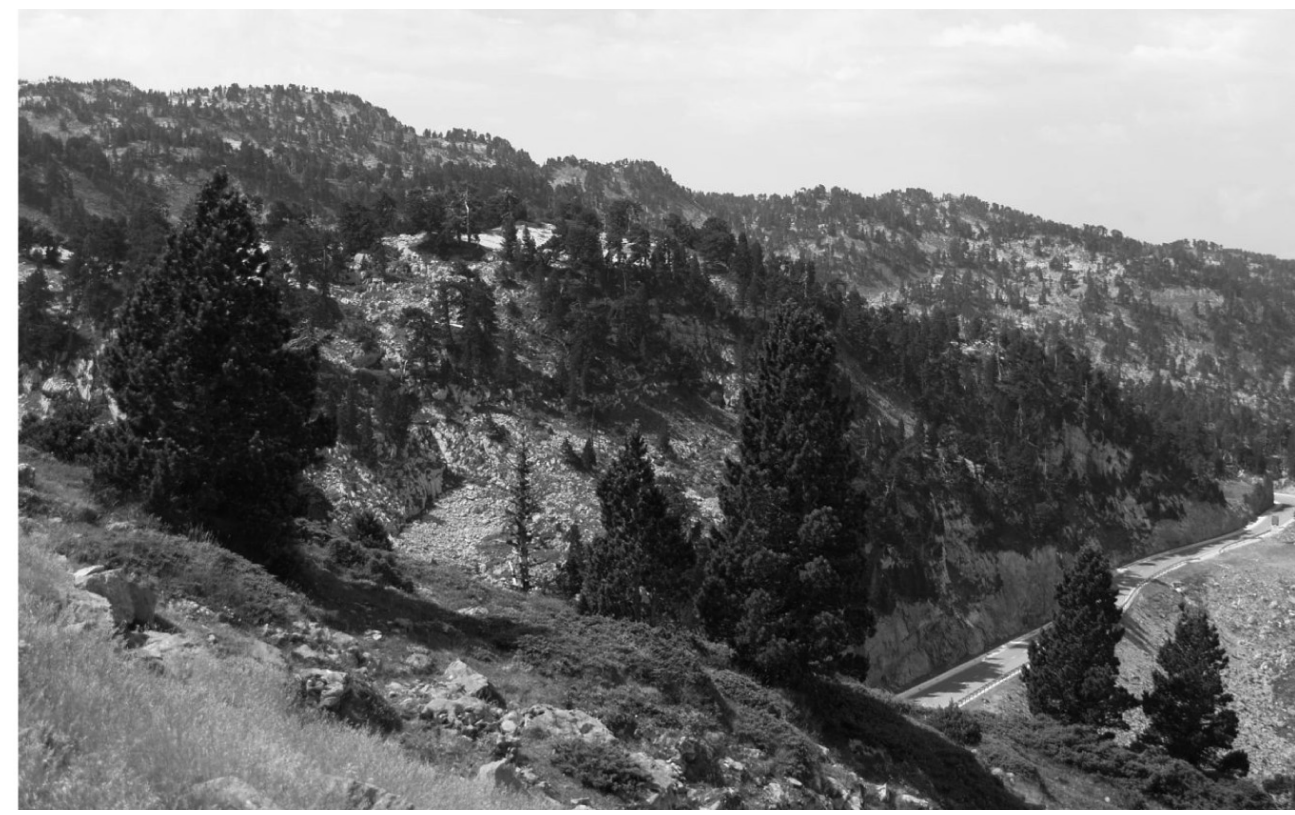

Imagen 1. Aspecto general del pinar de Pinus uncinata del Karst de Larra (Alto Pirineo Navarro-Suletino).

Las asociaciones inventariadas pertenecen a las dos facies que, en el piso subalpino del Pirineo Occidental, pueden diferenciarse en función, sobre todo, de la orientación y la insolación (Meaza, 1990; Loidi y Báscones, 1995). No obstante, existen otros dos factores que también determinan, aunque con menor protagonismo, cambios dentro de cada una de las facies: la pendiente y el grado de afloramiento de roca desnuda. Ambos suelen aparecer estrechamente interrelacionados, puesto que en aquellas parcelas o sectores con mayor pendiente se registra, a su vez, mayor cantidad de superficie rocosa. Por otra parte, estos dos factores aparecen íntimamente ligados al pastoreo, puesto que en 
las parcelas o sectores de escasa pendiente hay menos afloramientos de roca desnuda y, por tanto, son más frecuentados y presionados por la cabaña ovina, equina y vacuna, y por ungulados silvestres tales como el jabalí (Sus escrofa), corzo (Capreolus capreolus) y sarrio (Rupicapra pyrenaica). El pastoreo condiciona, a su vez, el grado de presencia y cobertura de especies herbáceas, pues escasean las menos palatables en tanto que cobran protagonismo las de mayor interés alimenticio (Festuca, Poa, Avenula...).

A continuación, se describe y caracteriza cada una de las facies y subfacies detectadas en el territorio de estudio:

- Los sininventarios 1 y 3 se tomaron en laderas orientadas al norte, y son encuadrables en el pinar esciófilo-quionófilo de Pinus uncinata a umbría con matorral de rododendro (Rhododendrum ferrugineum). Hay que hacer constar que el más paradigmático es el sininventario 1, mientras que el 3 representa una facies algo más empobrecida. No obstante, la diferencia a umbría no es tan amplia como la registrada a solana. De hecho, se basa más en valores globales de cobertura fundamentalmente del propio pino negro y, por tanto, mayor proporción de suelo o roca sin cobertura vegetal -caso del sininventario 3- que en un número menor de especies (34 y 37 taxones respectivamente).

- Los sininventarios 2 y 4 se obtuvieron en laderas orientadas al sur, y son asignables al pinar de heliófilo-quionófobo de Pinus uncinata a solana con matorral de gayuba (Arctostaphyllus uva-ursi) y enebro (Juniperus alpina). Ambos sininventarios son bastante prototípicos, aunque el 4 es considerablemente más pobre que el 2 tanto en especies como en cobertura.

El sininventario 5 responde a enclaves relativamente llanos con orientación TV. En este caso y como se ha señalado anteriormente, debido a la sobreexplotación ejercida por el ganado, nos encontramos ante agrupaciones vegetales bastante limitadas tanto en cobertura como en especies, muy similares a la versión pobre del sininventario 4.

Conviene señalar la importancia de un fenómeno altamente llamativo y no menos significativo que los autores de la presente aportación han podido constatar en los más de 20 años de estudio sobre el terreno, y muy especialmente en los 10 últimos: la creciente proliferación de pies de haya (Fagus sylvatica) en detrimento del pino en el cinturón ecotónico montano-subalpino (en torno a los 1650-1700 m de altitud). En las umbrías y, sobre todo, en las parcelas de suelo más profundo (vaguadas, fondos de dolina, recuencos kársticos...) la pujante haya gana progresivamente terreno al pino, que se ve obligado a acantonarse en roquedos y grietas de lapiaz. Así, mientras en nuestros primeros inventarios el haya era prácticamente inexistente, en los últimos años se ha detectado en casi una decena de ellos y siempre como pies jóvenes, de menos de 50 cm de porte; presentan, eso sí, una cobertura muy limitada y prosperan al bajo los pies de pino negro y al abrigo del rododendro. 


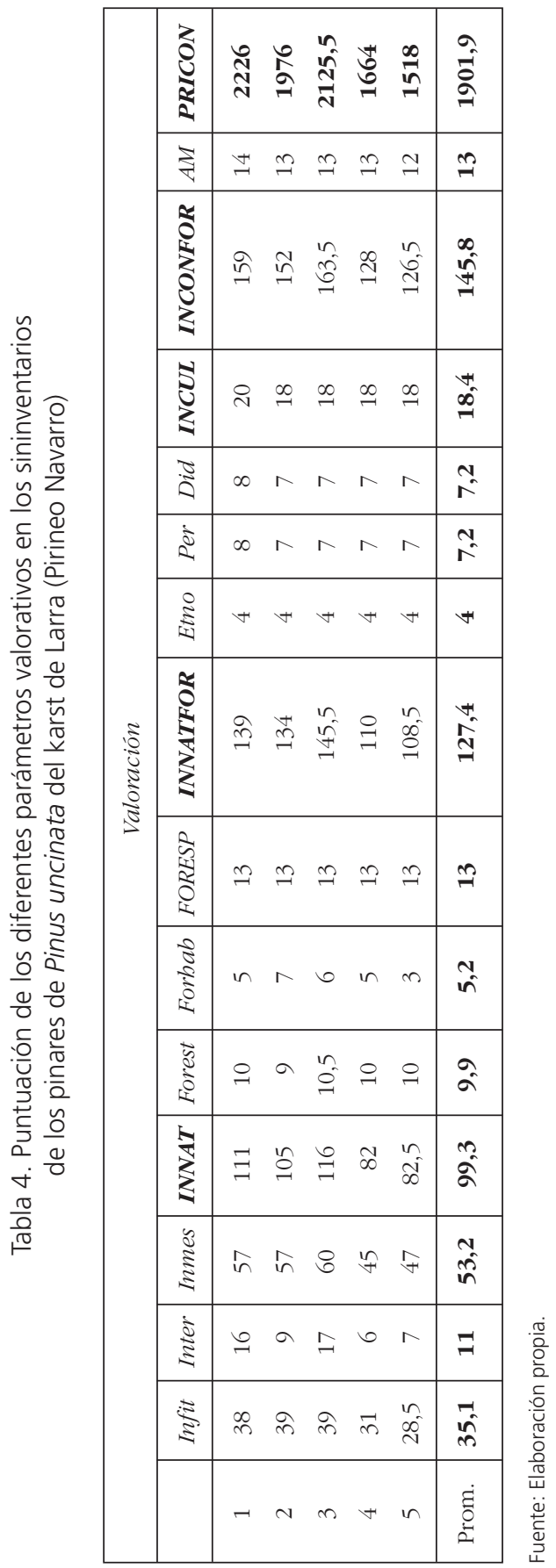


La tabla 4 muestra las diferentes puntuaciones parciales y totales del ejercicio evaluativo de los 5 sininventarios. Los aspectos más destacables de la clasificación final, correspondiente a la Prioridad de Conservación (PRICON) son los siguientes:

La mayor puntuación corresponde al sininventario 1, seguido del 3, el 2, el 4 y, por último, el 5. Los valores más altos correspoden a sininventarios de pinar de Pinus uncinata con matorral de Rhododendrum ferrugineum ( 1 y 3), con un promedio de 2175 puntos en el PRICON y de 161,25 en el INCONFOR; lo que es atribuíble:

- Sobre todo, al Interés Naturalístico y, dentro de éste, a los parámetros territoriales, puesto que los sininventarios 1 y 3 son más ricos en elementos raros ( 7 y 8 puntos respectivamente), endémicos (3 y 4 puntos) o finícolas (6 y 4 puntos) que el resto.

- También, a la alta diversidad y madurez del sininventario 1, que contribuyen a elevar la puntuación del INNAT.

- No tanto a los criterios mesológicos, puesto que al tratarse de sininventarios próximos y situados en un ambiente similar, aportan menos diferencias. Así, por ejemplo, como no podía ser de otra forma, la valoración de la función faunística es idéntica y máxima para todas, y casi equiparables las de la edáfica y la hidrológica. Se podría destacar, en su conjunto, la alta valoración que alcanza la parcela 3 en este aspecto.

Por contra, los sininventarios de pinar de Pinus uncinata con matorral de Arctostaphylos uva-ursi y Juniperus alpina (4 y 5, con un promedio de 1591 puntos en el PRICON y de 127,25 puntos en el INCONFOR) obtienen las puntuación aún más bajas, debido, sobre todo, a la escasa puntuación del sininventario 5, bastante parco en elementos territoriales valorables (es la parcela más pobre en taxones raros, endémicos o finícolas).

En lo que atañe a la metodología valorativa específicamente forestal, conviene indicar que, dado el carácter fragmentario y abierto de estos bosques, los índices menos discriminantes y, por ende, más problemáticos han sido los del cálculo del FORESP. Para acotarlo, hemos medido el área del conjunto del bosque en ortofotomapa mediante la herramienta "dibujar superficie a medir", de la página web del Gobierno de Navarra sobre fotografía y cartografía regional (2002: "http://sitna.cfnavarra.es/navegar"). Concretamente:

- Se ha asignado a todos los sininventarios la misma puntuación (13 puntos), correspondiente a una masa forestal de unas 46,54 ha que en la ortofoto comentada se percibe como homogénea, intercalada entre los hayedos montanos subyacentes y los pastizales y roquedos netamente subalpinos de cotas superiores.

- A pesar del carácter adehesado y escasa densidad arbórea de la generalidad de la superficie forestal, rasgo acentuado en muchos sectores por el pastoreo, se ha 
mantenido dicha puntuación conforme a su carácter esencialmente natural condicionado por factores litológico-climáticos.

- El hecho de que la carretera que enlaza Navarra con Francia atraviese la zona forestal no supone barrera alguna para la fauna y flora, puesto que el tráfico es habitualmente escaso, máxime en invierno, en que permanece cerrada por la nieve. Consecuentemente, no supone ninguna discontinuidad para contemplar la masa en su conjunto a la hora de valorar este criterio espacial.

En cuanto al FORFIS, todas las parcelas tienen valor 1, ya que la práctica totalidad de los fustes son exclusivamente de monte alto con porte forestal, normalmente monocaules. En lo que atañe al FORPAT, se ha asignado 3 puntos a cada una de las parcelas forestales por tratarse de una zona de alto interés arqueológico y etnográfico (pastoreo tradicional, presencia de elementos inmateriales y simbólicos destacados, caso del afamado "tributo de las tres vacas" representado por alcaldes roncaleses y bearneses en torno al mojón fronterizo de la Piedra de San Martín).

En la zona de estudio, a causa de la práctica inexistencia de explotación forestal y de la alta diversidad de ambientes y especies, la variable más diferenciadora entre sininventarios y que, por ello, se ha revelado como de sumo interés, ha sido la del FORHAB, que prima la diversidad de microhábitats espaciales contenidos dentro de un bosque o parcela, siempre que no sean segregables cartográficamente. Así, las puntuaciones oscilan entre los 7 puntos del sininventario 2, el más rico en microambientes (3 por diferentes hábitats rupícolas, 2 por la presencia de varios troncos añosos vivos o muertos en pie, 1 por la abundancia de troncos y ramas en el suelo, 1 por la abundancia y diversidad de líquenes epífitos y terrícolas), y los 3 del sininventario 5, también en este apartado la más exigua ( 1 por hábitat rupícola, 1 por tronco añoso vivo, 1 por tronco añoso caído).

Los índices de carácter estructural (FOREST), es decir los de "riqueza-diversidad de estratos" (RIQUEST) y "cobertura-densidad por estratos" (COBEST), no han supuesto apenas diferencias, pero se reafirman como de acotación ineludible.

Por último, el factor global de amenaza (AM), que se multiplica por el sumatorio de los valores antedichos (INCONFOR), tampoco fluctúa en exceso: supone 14 puntos para el sininventario 1; 13 para el 2, 3 y 4; y 12 para el 5. Su desglose muestra que la densidad demográfica es mínima ( 1 punto en cada uno de los cinco sininventarios), mientras que la accesibilidad-transitabilidad se presenta homogénea ( 6 para todos los sininventarios, salvo para el 5 , que obtiene 5 puntos). Las amenazas alternativas están relacionadas con la presión ejercida por la proliferación de visitantes durante todo el año (excursionistas, esquiadores, deportistas, turistas...); en general, alcanzan valores intermedios ( 6 puntos), un tanto al alza en el sininventario 1, ubicado en un área contigua a senderos y pistas de esquí de fondo. 


\section{Conclusiones}

Los resultados de la aplicación de nuestra propuesta metodológica muestran bien a las claras el notable interés geoecológico y alta prioridad de conservación de los pinares de Pinus uncinata del karst de Larra.

De un total de 27 inventarios biogeográficos realizados se han derivado 5 sininventarios, que muestran facies o subfacies bien diferenciadas y constituyen una buena representación de la vegetación forestal-frutescente del área subalpina de este sector pirenaico occidental. En concreto, se ha registrado un total de 68 especies vegetales en menos del 4\% total del territorio objeto de estudio. De ellas, 46 son herbáceas, 15 matas y trepadoras y tan sólo 7 árboles y arbustos.

Dos de los sininventarios (1 y 3), orientados al norte, son encuadrables en el pinar esciófilo-quionófilo de Pinus uncinata en umbría con matorral de rododendro (Rhododendrum ferrugineum). La diferencia entre ambos estriba más en los valores globales de cobertura vegetal -fundamentalmente del propio pino negro- y, por ende, de mayor o menor proporción de suelo o roca desnudos, que de número de especies.

Los sininventarios 2 y 4 , orientados al sur pueden asignarse al pinar heliófilo-quionófobo de Pinus uncinata en solana con matorral de gayuba (Arctostaphyllus uva-ursi) y enebro (Juniperus alpina). Ambos resultan bastante prototípicos, aunque el 4 es considerablemente más pobre tanto en coberturas generales como en especies (35 a 23). El sininventario 5 se asemeja en alto grado al 4, del que se diferencia por su exposición TV y uuna menor diversidad de especies a consecuencia de la sobreexplotación ejercida por los diferentes tipos de ganado.

En lo que respecta a la valoración global final (PRICON) de los diferentes sininventarios, existe una jerarquización clara: de mayor a menor, 1, 3, 2, 4 y 5 . En cuanto a valores intermedios, es el sininventario 3 el que presenta las puntuaciones parciales más altas, seguido del 1, 2, 4 y, siempre en última posición, el 5 (este último supone la versión o facies más pobre en todos los criterios de valoración registrados). La razón de que el sininventario 1 registre valores globales y finales superiores a los del 3 estriba en su mayor exposición a amenazas alternativas.

Finalmente, es interesante cotejar el valor global final (PRICON) más elevado obtenido en este sector del subalpino pirenaico (los 2226 puntos del pinar de Pinus uncinata del inventario 1) con los registrados por nuestro equipo de investigación en diversos ambientes forestales donde hemos tenido oportunidad de trabajar. En la Península Ibérica ninguna unidad analizada sobrepasa los 3000 puntos. Así, el robledal de Quercus pyrenaica llega a alcanzar los 1430, el quejigal de Quercus faginea 1380 y el encinar de Quercus rotundifolia 1365; siendo los valores más altos los del alcornocal de Quercus suber (2623,9; la puntuación más elevada en territorio europeo), tre- 
molinar de Populus tremula $(2384,5)$ y robledal de Quercus robur $(2383,5)$. El récord absoluto (superior a 3500 puntos) registrado hasta el momento por nuestro equipo corresponde al bosque con palma (Jubaea Chilensis) de la región chilena de Valparaíso.

\section{Bibliografía}

Cadiñanos, J.A. \& Meaza, G. (1998a) Bases para una Biogeografía aplicada. Criterios y sistemas de valoración de la vegetación. Geoforma ediciones, Logroño.

Cadiñanos, J.A. \& Meaza, G. (1998b) "Nueva propuesta metodológica de valoración del interés y de la prioridad de conservación de la vegetación". Actas del Colloque International de Botanique Pyreneo-Cantabrique. Mauleon.

Cadiñanos, J.A. \& Meaza, G. (2000) Metodología complementaria de evaluación de ecosistemas forestales. Inédito.

Cadiñano , J.A., Meaza, G. \& Lozano, P. (2002) "Valoración del interés y de la prioridad de conservación de bosques y comunidades preforestales de Larra (Alto Pirineo Navarro)". La Biogeografía: ciencia geográfica y ciencia biológica. Actas del II Congreso Español de Biogeografía. La Gomera.

Cadiñanos, J.A. et al. (2002) "Aplicación de una metodología de valoración de la vegetación a riberas fluviales: ensayo en el río Butrón (Bizkaia)". Aportaciones geográficas en memoria del Prof. L. Miguel Yetano Ruiz, 6588. Zaragoza.

Cadiñanos, J.A., Lozano, P. \& Quintanilla, V. (2011) "Propuesta de marco metodológico integrado para la valoración biogeográfica de espacios Red Natura 2000 de la comunidad autónoma del País Vasco. El ejemplo de Gárate-Santa Bárbara (Guipúzcoa)”. Boletín de la AGE 57: 33-56. Madrid.
Kirby, K. (1986) Forest and Woodland evaluation. En Wildlife Conservation Evaluation: 202-221, Chapman and Hall. London.

Loidi, J. y Bascones, J.C. (1995) Mapa de Series de vegetación de Navarra a escala 1:200.000, $y$ memoria. Gobierno de Navarra. Pamplona.

Lorda, M. (2001) Flora del Pirineo navarro. Guineana $7,1-557$.

Lozano, P. J., Cadiñanos, J.A., Longares, L.A. \& Cid, M.A. (2007) "Valoración Biogeográfica de los tipos de bosque en la combe de Huidobro (Parque Natural de las Hoces del Ebro-Burgos)". Actas del $4^{\circ}$ Congreso Español de Biogeografía, Ávila.

Meaza, G. (1990) El paisaje vegetal del Alto Pirineo Navarro. Lurralde 13, 207-238. Donostia-San Sebastián.

Meaza, G. (1993) "Propuesta metodológica de valoración fitogeográfica de unidades de paisaje vegetal". Libro Homenaje al investigador Felix M. Ugarte Elorza, 369-391. Bilbao.

Meaza, G y Cadiñanos, J.A. (2000) "Valoración de la Vegetación". En "Metodología y Práctica de la Biogeografia". Ediciones del Serbal, Barcelona.

Meaza, G., Cadiñanos, J.A. \& Lozano, P. (2006) "Valoración biogeográfica de los bosques de la reserva de la biosfera de Urdaibai (Vizcaya)". Actas del III Congreso Español de Biogeografía. Reserva de la Biosfera de Urdaibai. 
Peralta, J. (2005) Hábitats de Navarra de interés y prioritarios (Directiva Hábitats). Servicio de Publicaciones. Universidad Pública de Navarra. Pamplona.
Quintanilla, V., Cadiñanos, J.A., Latasa, I. y Lozano, P.J. (2012) "Estudio Biogeográfico de los bosques del área mediterránea de Chile: Inventario y Caracterización." Boletín de la $A G E$ 60: 91-114. Madrid. 